OPEN ACCESS

Edited by:

Marc N. Potenza,

Yale University, United States

Reviewed by:

Melanie L. Schwandt,

National Institutes of Health (NIH),

United States

Li Shen,

University of Southern California,

United States

*Correspondence:

Mengfan Xia

mengfanxia513@126.com

†These authors have contributed equally to this work

Specialty section: This article was submitted to

Addictive Disorders,

a section of the journal

Frontiers in Psychiatry

Received: 17 October 2020 Accepted: 28 May 2021

Published: 09 July 2021

Citation:

Yang C, Xia M, Li T and Zhou Y (2021)

How Do Specific Social Supports

(Family, Friend, and Specialist) Reduce

Stress in Patients With Substance Use

Disorders: A Multiple Mediation

Analysis. Front. Psychiatry 12:618576.

doi: 10.3389/fpsyt.2021.618576

\section{How Do Specific Social Supports (Family, Friend, and Specialist) Reduce Stress in Patients With Substance Use Disorders: A Multiple Mediation Analysis}

\author{
Chunyu Yang ${ }^{1 \dagger}$, Mengfan Xia ${ }^{2 *}$, Tianshu $\mathrm{Li}^{3+}$ and You Zhou ${ }^{4}$ \\ ${ }^{1}$ Nanjing University of Information Science and Technology, Research Institute of Climatic and Environmental Governance, \\ Institute of Prison Sociology and Anthropology, College of Law and Political Science, Nanjing, China, ${ }^{2}$ Department of \\ Sociology, Nanjing University, Nanjing, China, ${ }^{3}$ Faculty of Arts and Social Science, The University of Sydney, Sydney, NSW, \\ Australia, ${ }^{4}$ The University of Melbourne, Parkville, VIC, Australia
}

Perceived social support has been found to reduce the stress of individuals who suffer from substance use disorders. However, the mediating effects of resilience and affect balance in the relationships between specific social supports (family, friend, and significant others) and perceived stress are still unclear. This study focused on substance use disorders (SUD) patients, exploring the mediating roles of resilience and affect balance on the relationships between three dimensions of social supports (family, friend, and specialist) and stress. Three hundred thirty-nine participants completed questionnaires of perceived social support, resilience, affect balance, and stress. After controlling resilience and affect balance, the results suggested the effects of perceived family and specialist supports on perceived stress were fully mediated, and the association between perceived friend support and perceived stress is partially mediated. The multiple mediation analysis showed resilience is significant in mediating the relationship between specific perceived supports in all models, while affect balance is only significant in mediating the relationship between specialist support and perceived stress. Implications for enriching current theoretical research and strategies for government and practitioners were also discussed.

Keywords: perceived social support, resilience, affect balance, stress, patients with SUD

\section{INTRODUCTION}

Substance abuse disorder is a worldwide public health problem. It has brought many social stressors on patients with SUD, such as unemployment, stigmatization, and discrimination (1), forming a vicious circle of social isolation, which in turn increases the risk of relapse (2). In addition, stress may overwhelmingly lead to individuals' physical and psychological disorders, such as depression (3), cognitive function impairment (4), cardiovascular disease, and even mortality (5). Therefore, it is theoretically and practically essential to explore the possible methods of reducing stress levels in individuals who abuse substances.

\section{Perceived Stress and Perceived Social Support}

Perceived stress refers to the perceived contradictions that external and internal demands exceed individuals' adaptability (6). Stress is not an inherent issue. Instead, it depends on whether 
individuals have sufficient capacity and social networks to cope with. Numerous studies have denoted that perceived social support is beneficial for health-promoting behaviors that protect individuals from the negative effects of stressful life events $(7,8)$. Perceived social support is conceptualized as available resources and assistance given by accessible individuals or groups that may help individuals address stressful events (9).

The cognitive-transactional stress theory (10) denotes that perceived stress is a dynamic cognitive assessment process, including potential stress events, primary cognitive assessment of events, secondary assessments, coping style choices, and outcomes of adaptation. Individuals' assessment and cognition of stress determine the choice of individual coping styles. An individual's ability to address stress depends on the individual's belief that there are sufficient resources and social support to counteract the harmful impacts of stress. People with high social support tend to experience better mind and body conditions, which are conducive to enhancing self-confidence, providing supports for reducing personal stress (11). Empirical studies show that perceived social support helps individuals overcome the adverse impacts of stressful events that improve their social adaptability (12). Given dimensions of social support (i.e., relationship-specific support) are distinct constructs, it is recommended to study the roles of three types of social supports (family, friend, and specialist supports) (13). From empirical perspectives, unmasking the underlying relationship between different dimensions of social support and stress in patients with SUD has enormous significance in updating the knowledge and measurements regarding designing specific treatments and interventions to reduce SUD individuals' stress and promote their integral well-beings. Therefore, the current study was designed for investigating the potential mediation roles of resilience and affect balance between three subtypes of perceived social support and stress in individuals with SUD. Based on the theoretical and empirical literature, it is expected to propose the first hypothesis of the current study:

H1. Specific perceived social supports (family, friend, and specialist) have a significant and negative direct effect on perceived stress;

\section{Perceived Social Support, Resilience, Affect Balance, and Stress}

Resilience is defined as a trait that is beneficial for buffering the detrimental effects of stressors and adversities and promoting positive bio-psycho-social functions (14). Resilience is a resource and asset of an individual, which can help deal with adverse conditions and make rational choices, generating positive outcomes (15). Recently, an increasing number of scholars have been paying attention to the protective factors embedded in individuals' social environment, aiming to help individuals utilize various active resources to promote ideal results (16). Family, peer groups, and important others are crucial social capital for individual development, especially drug users. Wang et al. (17) suggest that social support is an essential external protective factor that can promote resilience. Drug users with sufficient social networks can adjust themselves more effectively and efficiently when facing adversity and difficulties $(7,18)$. Also, Cai et al. (19) has suggested that resilience is a mediator between perceived social support and perceived stress. Individuals owning high levels of perceived social support tend to obtain assistance from their social network, which transforms the social capital into psychological capital, enhancing the ability to bounce back while confronting stressful events (20). Based on the antecedent literature, it is rational to propose the second hypothesis of the current study:

H2. Resilience mediates the associations between specific perceived social supports (family, friend, and specialist support) and stress;

Affect balance may be another potential mediator of the association between specific perceived social supports and perceived stress. Affect balance denotes the capability of balancing positive and negative affections. People with higher scores of affect balance have more positive affect than negative affect (21). Studies have identified perceived social support is a significant predictor of affect balance (22). For example, prior research suggests that perceived social support is positively correlated with affect balance and self-esteem (23) and negatively correlated with loneliness among SUD patients (24). Further, some research suggests that affect balance is negatively associated with stress $(25,26)$. Based on the broaden-and-build theory (27), positive emotions can help individuals address challenges with a positive attitude, broaden their thinking, expand their perceptions, and choose more creative and flexible actions, while negative emotions often narrow individuals' abilities. The theory holds that affect balance can activate actions, expand awareness, buildings resources, and relieve stress (27). Stroud et al. (28) outlined a physiological model that affective states are significantly associated with individuals' health and well-being. Poor affect balance is closely linked with a high level of perceived stress. In a word, individuals with higher perceived social support tend to make better use of the surrounding environment and, in turn, enhance their performance of affect balance, helping them to cope with stress (29). Given the findings from theoretical and empirical studies, it is expected to propose the third hypothesis:

H3. Affect balance mediates the associations between specific perceived social supports (family, friend, and specialist support) and perceived stress;

In addition, it should be noted that resilience and affect balance are not independent mediators. Substantial studies have suggested that resilience can promote affect balance (30-32). It has been shown that individuals with a higher level of resilience can deal with their emotional conflicts more successfully (33-35). Therefore, it is expected to assume that perceived social support impacted stress through serial-mediation effects of resilience and affect balance. Therefore, we proposed the fourth hypothesis regarding serial mediations:

H4. The serial mediation path of perceived social support (family, friend, and specialist support) $\rightarrow$ resilience $\rightarrow$ affect balance $\rightarrow$ perceived stress is significant; 


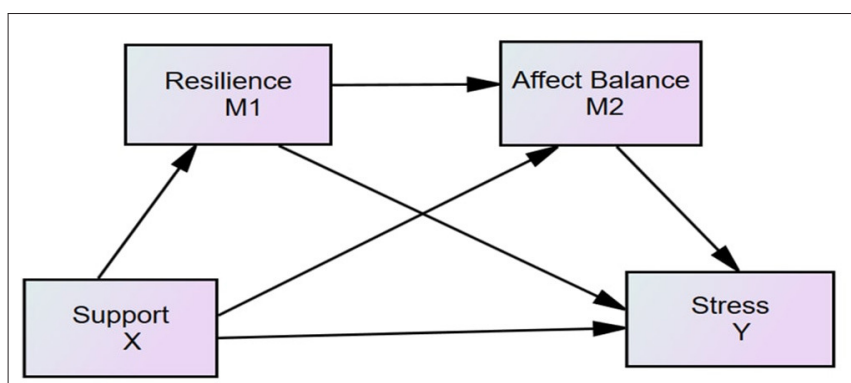

FIGURE 1 | Hypothesized model. X, independent variable; Y, dependent variable; M1, the first mediator; $M 2$, the second mediator; Support, family/friend/specialist/supports; Stress, perceived stress.

\section{The Current Study}

To our knowledge, although there are numerous studies on the relationship between specific perceived social supports and stress, there is no research that simultaneously investigates the relationships between specific social supports and stress, especially among patients with SUD. Based on the antecedent literature, the current study proposed three models, examining the effects of specific perceived social supports (family, friend, and specialist, respectively), on perceived stress in individuals diagnosed with severe SUD addiction level. The hypothesized model is shown in Figure 1.

\section{METHOD}

\section{Participants and Procedure}

All patients (256 males and 83 females) who volunteered to participate in the study were from two compulsory rehabilitation centers in a southern city of China. They were informed of the detailed information of the research project and confidentiality before joining the project. The whole survey took about $30 \mathrm{~min}$, during which participants were allocated separately in independent conference rooms. All 339 patients were from 20 to 60 years old [mean age $=38.64$ years, standard deviation (S.D.) $=9.10$ years]. $69.3 \%$ of the participants were relapsers in the sample, and $30.7 \%$ were getting their first treatment. For the drug consumption history, $18.4 \%$ of the participants used drugs $<5$ years, $30.2 \%$ of the participants took drugs from 5 to 10 years, and $51.4 \%$ took drugs more than 10 years. For the last time of taking illicit drugs, $17.4 \%$ of the participants were 1 month ago, $23.1 \%$ of the participants were $1-3$ months ago, $16.5 \%$ of the participants were 3-6 months ago, and $42.5 \%$ of the participants were more than half a year ago. The detailed demographic information is shown in Table 1.

\section{Measures}

\section{Perceived Social Support}

Multi-dimensional Scale of Perceived Social Support (MSPSS) was implemented to measure perceived social support. MSPSS has three subscales: perceived family support subscale, perceived friends support subscale, and perceived specialists support subscale. Participants were requested to rate this 7 -point scale from $1=$ "strongly disagree" to $7=$ "strongly agree" (36). The scores of specific perceived social supports were added up by all items in every subscales. A higher score represents a higher level of specific perceived social support. The scale of MSPSS has shown satisfactory consistency and is widely applied among Chinese groups (37). In the present study, Cronbach's alpha of the perceived family, friend, specialist supports were 0.858 , $0.825,0.821$, which suggests that all subscales of perceived social supports have good reliability.

\section{Resilience}

The Connor-Davidson Resilience Scale (CD-RISC) has 25 items used to measure the ability to deal with adversity. Participants were asked to rate on CD-RISC based on their perception of each item over the last month (38). The scale consists of items such as "I can adapt to change," "I have a close and safe relationship," and "No matter what happens, I can handle it." CD-RISC is a four-point Likert scale $(0=$ "none at all," $4=$ "almost always"), with a total score from 0 to 100 . Higher scores represent greater resilience. Previous studies suggest that the Chinese version of the CD-RISC indicated excellent reliability $(39,40)$. The Cronbach's alpha of CD-RISC was 0.900, which suggests that CD-RISD has very good reliability in the current study.

\section{Affect Balance}

Affect balance was assessed with the Positive and Negative Affect Scale (PANAS) designed by Watson et al. (41). The questionnaire consists of 20 items, including two subscales of positive affect (10 positive affections, e.g., "interested" and "enthusiastic") and negative affect (10 negative affections, e.g., "guilty" and "distressed"). PANAS is a 5-points scale evaluating individuals' positive and negative feelings over last few weeks $(1=$ very slight or not at all, $2=$ relatively small, $3=$ moderate, $4=$ considerable, $5=$ very strong). The score was calculated by subtracting the sum of negative affect items from the sum of positive affect items (42). Previous studies have shown that the PANAS in Chinese indicates good reliability and validity (24). The Cronbach's alpha of PANAS was 0.890 , indicating good reliability in the current study.

\section{Perceived Stress}

This study applied the Perceived Stress Scale (PSS) for measuring the perceived pressure of the patients. The PSS is a 14-item scale in which participants can rate their perceptions regarding how much stress they have experienced over the last 4 weeks (43). PSS has four points 4-points scale, with response value ranging from 0 (never) to 4 (frequent). A higher score reflects a higher level of perceived stress. The PSS-14 scale has revealed high reliability in the Chinese context (44). Cronbach's alpha of PSS was 0.706, indicating acceptable reliability. The values of Cronbach's alpha of all measured scales indicated at least the acceptable reliability standard for the following mediation analysis.

\section{Addiction Severity Diagnostic Questionnaire (DSM-5)}

This study implemented a DSM-5-based self-reported questionnaire to measure the addiction severity of the participants. The DSM-5 addiction severity is measured by evaluating 11 symptoms/criteria on the subjects (45). The symptoms include (1) craving; (2) tolerance; (3) hazardous use; 
TABLE 1 | Sample characteristics.

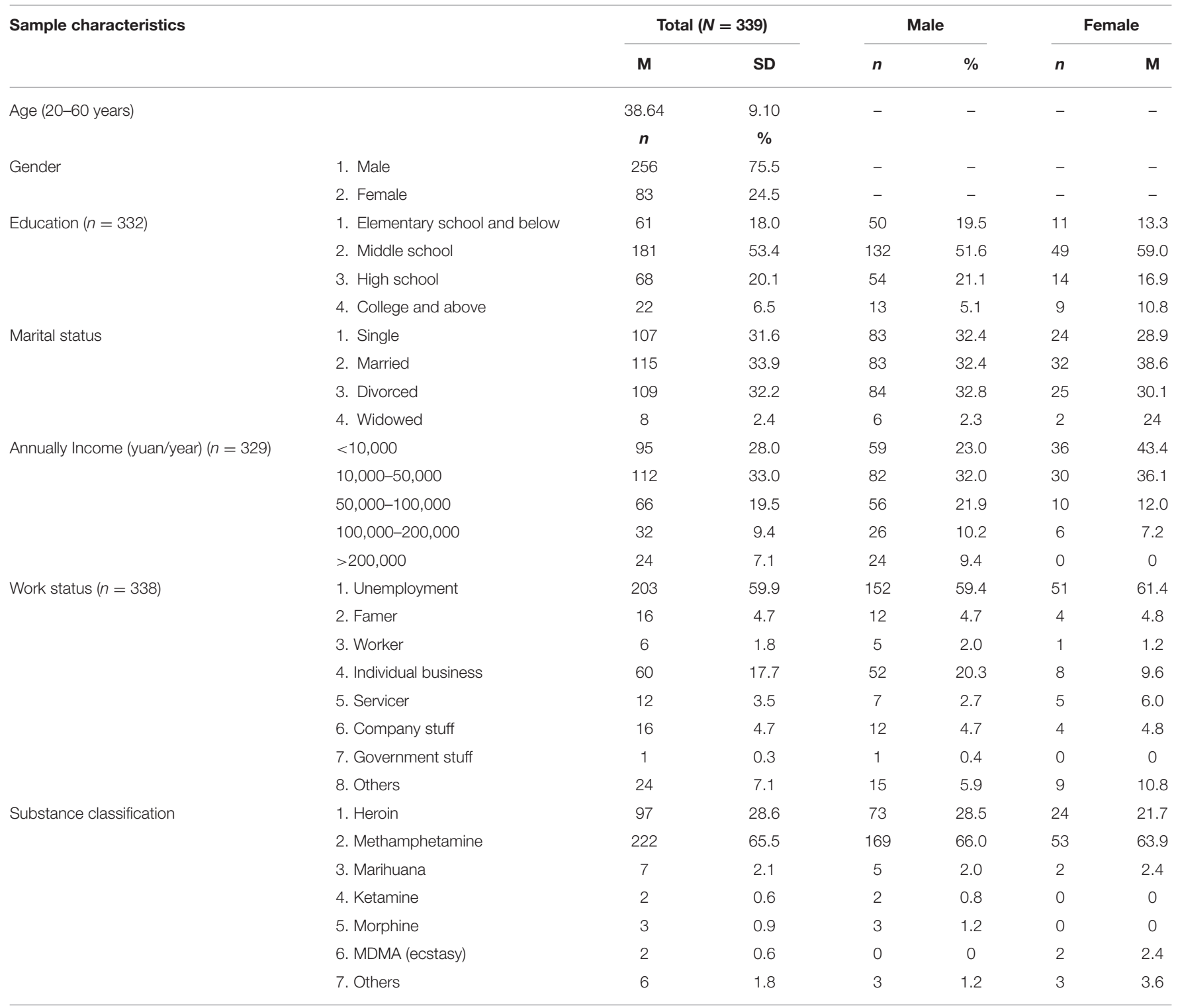

(4) withdrawal; (5) prolonged use of substantial amounts; (6) collapse of relational and social connections; (7) withdrawal from social and occupational events; (8) use-related physical and psychological issues; (9) substantial using time; (10) social and interpersonal problems related to use; (11) repeated attempts of abstinence (45). There are three levels of addiction severity which are measured by counting the symptoms/criteria: 2 to 3 is mild level, 4 to 5 is moderate level, 6 and above is severe level (45). The results showed that all 339 participants were diagnosed with severe addiction. The primary reason was that they accepted abstinence treatment in a compulsory rehabilitation center, which is a mandatory drug treatment center specializing in curing patients with chronic SUD. In this study, its Cronbach's alpha was 0.730 .

\section{Data Analyses}

In this study, IBM SPSS version 22 was utilized to analyze the descriptive statistics. Pearson analysis was operated to examine the bivariate correlation between social supports (family, friend, and specialist supports), resilience, affect balance, and stress. Then, we created serial multiple mediation models to investigate the mediation effects of resilience and affect balance on the association between family, friend, specialist social supports, and perceived stress. The bootstrapping in SPSS PROCESS macro was used to test the mediation effects of the study (46). By random sampling, 10,000 samples were generated and employed the $95 \%$ confidence interval (CI) in the analysis of the mediation effects (47). If lower and upper bounds of 95\% CI do not span zero, the path is significant at the 0.05 level. The analyses were administered for all models. 
TABLE 2 | Means, standard deviations (SD), reliabilities and inter-correlations among study variables.

\begin{tabular}{|c|c|c|c|c|c|c|c|c|c|c|}
\hline Number & Measure & Mean & SD & Alpha & 1 & 2 & 3 & 4 & 5 & 6 \\
\hline 1 & Family support & 18.45 & 5.45 & 0.858 & 1 & & & & & \\
\hline 2 & Friend support & 16.52 & 5.13 & 0.825 & $0.495^{\star \star}$ & 1 & & & & \\
\hline 3 & Specialist support & 17.35 & 5.56 & 0.821 & $0.745^{\star \star}$ & $0.570^{\star \star}$ & 1 & & & \\
\hline 4 & Perceived social support & 52.33 & 14.08 & 0.906 & $0.874^{\star \star}$ & $0.796^{\star \star}$ & $0.912^{\star \star}$ & 1 & & \\
\hline 5 & Resilience & 76.67 & 16.64 & 0.900 & $0.402^{\star \star}$ & $0.426^{\star \star}$ & $0.502^{\star \star}$ & $0.523^{\star \star}$ & 1 & \\
\hline 6 & Affect balance & 1.70 & 7.15 & 0.890 & $0.221^{\star \star}$ & $0.201^{\star \star}$ & $0.291^{\star \star}$ & $0.278^{\star \star}$ & $0.300^{\star \star}$ & 1 \\
\hline 7 & Stress & 41.20 & 5.64 & 0.706 & $-0.130^{\star \star}$ & $-0.278^{\star \star}$ & $-0.282^{\star \star}$ & $-0.231^{\star \star}$ & $-0.386^{\star *}$ & $-0.313^{\star \star}$ \\
\hline
\end{tabular}

$\alpha=$ Cronbach's alpha.

${ }^{* *}$ significant at the 0.01 level (2-tailed).

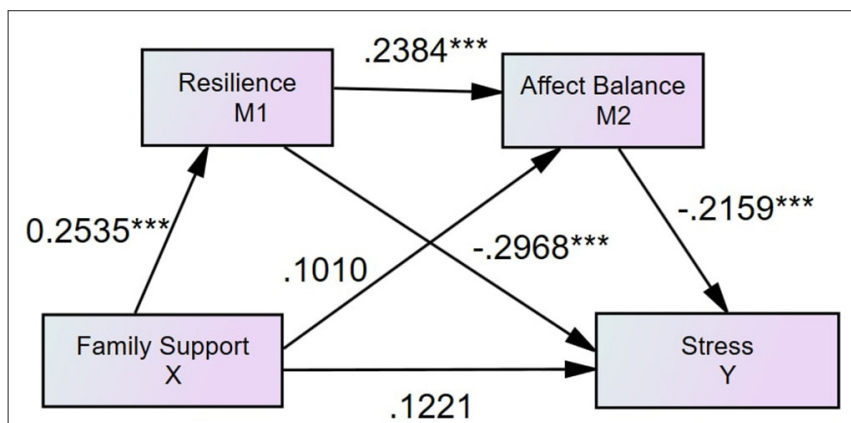

FIGURE 2 | Standardized structural model (family support, $N=339, R^{2}=$ 0.2188). ${ }^{* * *} p<0.001$

\section{RESULTS}

\section{Preliminary Analyses}

Overall, we calculated the means, standard deviations (S.D.), Cronbach coefficients, and bivariate correlations (see Table 2). The results suggested all variables were significantly correlated.

\section{Family Support, Resilience, Affect Balance, and Perceived Stress}

To evaluate the independent effects of family support, friend support, and specialist support were controlled as covariates in this serial mediation model. The results showed that resilience mediates the association between perceived family support and perceived stress (see Figure 2, Table 3). The direct path from family support to perceived stress $(\beta=0.1221, p=0.3949)$ and the indirect path from family support to stress via affect balance was insignificant ( $\beta=-0.0259,95 \% \mathrm{CI}=[-0.0735,0.0123])$. The indirect path from family support to perceived stress via resilience $(\beta=-0.0894,95 \%$ CI $=[-0.1778,-0.03626])$ was significant.

\section{Friend Support, Resilience, Affect Balance, and Perceived Stress}

After controlling family support and specialist support as the covariates, the results also demonstrated that resilience mediated the relationship between perceived friend support and stress (see Figure 3, Table 3). The direct effect of perceived friend support on stress was statistically significant $(\beta=-0.1942, p=0.0014)$. Moreover, the indirect path from friend support to stress via resilience was significant $(\beta=-0.0762,95 \% \mathrm{CI}=[-0.1570$, $-0.0248])$, but via affect balance $(\beta=-0.0033,95 \% \mathrm{CI}=$ $[-0.0453,0.0407])$ was insignificant.

\section{Specialist Support, Resilience, Affect Balance, and Perceived Stress}

After controlling family support and friend support as the covariates, the results found that the association between specialist support and perceived stress is mediated by resilience and affect balance (see Figure 4, Table 3). The direct path from specialist support to stress was insignificant $(\beta=0.1076$, $p=0.1873$ ), and the path from specialist support to stress via resilience $(\beta=-0.1525,95 \% \mathrm{CI}=[-0.2917,-0.0570])$ and via affect balance $(\beta=-0.0486,95 \% \mathrm{CI}=[-0.1268,-0.0042])$ were significant.

\section{Serial Mediation}

The results showed that the total indirect effects of family support $(\beta=-0.0115,95 \% \mathrm{CI}=[-0.0232,-0.0007])$, friend support $(\beta$ $=-0.0108,95 \% \mathrm{CI}=[-0.0258,-0.0022])$, specialist support $(\beta=-0.0216,95 \% \mathrm{CI}=[-0.0494,-0.0053])$ on perceived stress through resilience and affect balance were significant (see Table 3).

\section{DISCUSSION}

The current study systematically analyzed the underlying relationship between specific perceived social supports (family, friend, and specialist, respectively), and perceived stress by examining the mediating effects of resilience and affect balance among SUD patients. It turned out that only perceived friend support has a direct effect on perceived stress among SUD patients. Moreover, resilience and affect balance fully mediate the associations between perceived family and specialist supports and perceived stress. In contrast, resilience partially mediates the association between perceived friend support and perceived stress. We found that the serial mediations, specific perceived social supports (friend, family, specialist) $\rightarrow$ resilience $\rightarrow$ affect balance $\rightarrow$ perceived stress, were significant in all models. The present study's findings implied that three types of 
TABLE 3 | Bootstrapping indirect effects and 95\% confidence intervals (Cl) for the final mediation model.

\begin{tabular}{|c|c|c|c|}
\hline \multirow[t]{2}{*}{ Model pathways } & \multirow[t]{2}{*}{ Point estimates } & \multicolumn{2}{|c|}{$95 \% \mathrm{Cl}$} \\
\hline & & Lower & Upper \\
\hline \multicolumn{4}{|l|}{ Total indirect effect } \\
\hline Family support $\rightarrow$ Stress & -0.1308 & -0.2183 & -0.0588 \\
\hline Friend support $\rightarrow$ Stress & -0.0903 & -0.1799 & -0.0185 \\
\hline Specialist support $\rightarrow$ Stress & -0.2227 & -0.3817 & -0.1037 \\
\hline Perceived social support $\rightarrow$ Stress & -0.1064 & -0.1650 & -0.0595 \\
\hline \multicolumn{4}{|l|}{ Indirect effect } \\
\hline Family support $\rightarrow$ Resilience $\rightarrow$ Stress & -0.0894 & -0.1778 & -0.0326 \\
\hline Family support $\rightarrow$ Resilience $\rightarrow$ Affect balance $\rightarrow$ Stress & -0.0155 & -0.0335 & -0.0043 \\
\hline Family support $\rightarrow$ Affect balance $\rightarrow$ Stress & -0.0259 & -0.0672 & 0.0123 \\
\hline Friend support $\rightarrow$ Resilience $\rightarrow$ Stress & -0.0762 & -0.1570 & -0.0248 \\
\hline Friend support $\rightarrow$ Resilience $\rightarrow$ Affect balance $\rightarrow$ Stress & -0.0108 & -0.0258 & -0.0022 \\
\hline Friend support $\rightarrow$ Affect balance $\rightarrow$ Stress & -0.0033 & -0.0453 & 0.0407 \\
\hline Specialist support $\rightarrow$ Resilience $\rightarrow$ Stress & -0.1525 & -0.2917 & -0.0570 \\
\hline Specialist support $\rightarrow$ Resilience $\rightarrow$ Affect balance $\rightarrow$ Stress & -0.0216 & -0.0494 & -0.0053 \\
\hline Specialist support $\rightarrow$ Affect balance $\rightarrow$ Stress & -0.0486 & -0.1268 & -0.0042 \\
\hline Perceived social support $\rightarrow$ Resilience $\rightarrow$ Stress & -0.0783 & -0.1383 & -0.0347 \\
\hline Perceived social support $\rightarrow$ Resilience $\rightarrow$ Affect balance $\rightarrow$ Stress & -0.0113 & -0.0245 & -0.0028 \\
\hline Perceived social support $\rightarrow$ Affect balance $\rightarrow$ Stress & -0.0169 & -0.0312 & -0.0044 \\
\hline
\end{tabular}

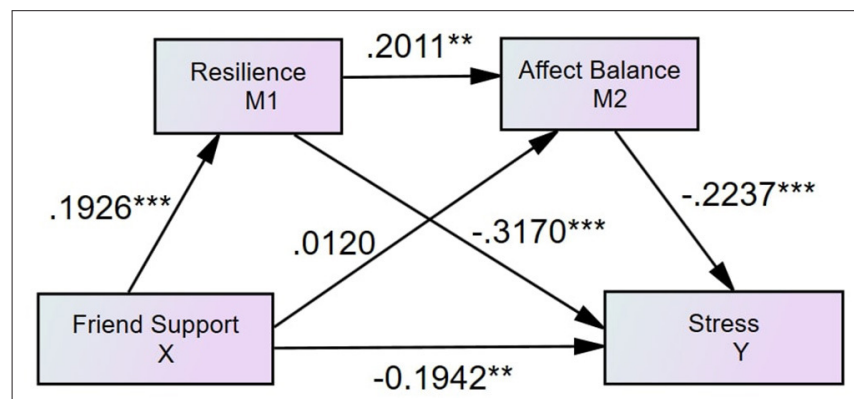

FIGURE 3 | Standardized structural model (friend support, $N=339, R^{2}=$ 0.0820). ${ }^{* * *} p<0.001,{ }^{* *} p<0.01$.

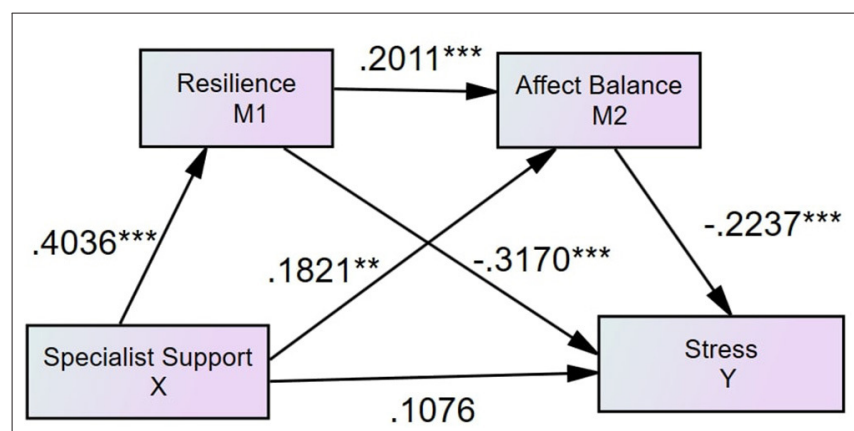

FIGURE 4 | Standardized structural model (specialist support, $N=339, R^{2}=$ 0.2188). ${ }^{* * *} p<0.001,{ }^{* *} p<0.01$.

perceived social supports, resilience, and affect balance are critical protective factors for SUD patients to reduce perceived stress.

Social support is generally considered as the effective caring from specific social networks (11). Family, friends, and specialist supports are three types of perceived social supports (36). The current study's findings showed that perceived social support's direct effects on perceived stress might vary by support types. Contrary to Hypothesis 1, the findings suggest that only perceived friend support directly affects perceived stress in SUD patients, which is inconsistent with the previous findings (48). One possible explanation of the insignificant direct effect of perceived family support on perceived stress is that the mediation effects of resilience and affect balance are strong enough to fully mediate the effect of perceived family support on perceived stress. Similarly, the explanation for the insignificant direct effect of perceived specialist support can also be attributed to the strong mediation effects of resilience and affect balance. However, the full mediations do not mean that perceived family support and perceived specialist support are not effective in reducing perceived stress among SUD patients. The $R^{2}$ of family support and specialist support models are both 0.22 (see Figures 2, 4), suggesting that perceived family and specialist support exert significant effects on decreasing perceived stress through the mediators of resilience and affect balance that performs full mediation effects. Thus, it is recommended to design and develop support-based interventions for SUD patients. Future research is expected to explore how to design the relevant projects to boost family, friend and specialist support for SUD patients.

Consistent with Hypothesis 2, the findings suggested resilience mediates all the effects of three types of social supports on perceived stress among SUD patients, which aligns with the study conducted on Chinese soldiers (19). In particular, the findings suggest that resilience fully mediates the impacts of perceived family support and perceived specialist support on perceived stress in people with SUD. The findings were 
in line with the study conducted by Wu et al. (49) that the support provided by family members is a significant predictor of resilience. Moreover, the findings suggested that friend support can, directly and indirectly, reduce perceived stress (50). The support of peers can give SUD patients a sense of belonging and satisfy their need for recognition in society (51). Wolin and Wolin (52) also found that stable relationships with their peers and family members are conducive to the formation of resilience for SUD patients, enhancing confidence in coping with stress. The theoretical underpinning is that people who have adequate psychological capital and diversities of coping strategies are more likely to gain resilience, which is beneficial for reducing stress (53). Although the current research found that perceived friend support, directly and indirectly, affects perceived stress, it did not suggest that family support and specialist support are not as vital as friend support regarding reducing SUD patients' stress. Instead, the variances that family support model $\left(R^{2}=\right.$ 0.22 , see Figure 2) and specialist support model $\left(R^{2}=0.22\right.$, see Figure 4) account for patients' stress reduction are higher than the variance that is explained by the friend model $\left(R^{2}=\right.$ 0.08 , see Figure 3 ). Therefore, it indicated that rehabilitation centers are recommended to wisely and dynamically incorporate family, friend, and specialist supports during rehabilitation phases. Future studies may further investigate the effectiveness of interventions designed for developing family, friend and specialist supports.

Partially consistent with Hypothesis 3, the findings revealed that the associations between specialist support and perceived stress were mediated by affect balance. The findings corresponded with existing research conducted in other demographic groups (22). However, the paths from perceived family support and friend support to affect balance are insignificant. Based on the social learning theory (54), behaviors can be learned from social observation and interaction. As the study was conducted in compulsory rehabilitation centers, the detained individuals are obstructed to learn affect balance skills through the interaction processes with their families and friends. Overall, the findings implied that a higher level of support from specialist improves SUD patients' affect balance which in turn decrease their perceived stress.

The results further validate Hypothesis 4, which indicated that three serial mediation (family, friend, specialist supports $\rightarrow$ resilience $\rightarrow$ affect balance $\rightarrow$ perceived stress) were significant in all models. The findings indicated that resilience mediated the association between specific perceived social supports and affect balance. The findings also revealed that affect balance acted as a mediator of the association between resilience and perceived stress, which is in line with previous studies that SUD patients with a high perception of social support are prone to show more resilience (44). Precious studies suggest that those with higher resilience are more likely to experience higher affect balance (55), which in turn decreases perceived stress (56). The theoretical underpinning of the current findings is that different dimensions of perceived social support may promote resilience, while resilient individuals are good at balancing their affections, ultimately reducing stress perception.

\section{LIMITATIONS AND CONCLUSIONS}

However, this research also has some limitations that should be considered. First, the data of the study were collected from self-reported scales. Due to the limitations of self-report data, it is recommended to apply multiple assessment methods in future studies, such as collecting data from parents, communities, and rehabilitation center databases. Second, cross-sectional data implemented in this study cannot explore the causal relationship between variables. In further research, it is recommended to use mixed methods, such as longitudinal and experimental methods. Third, the current findings are only focused on the population of SUD patients with severe addiction level from compulsory rehabilitation centers, in which the findings may only apply to the individual with similar contexts. Future research is expected to have other groups, such as the SUD groups with mild and moderate addiction levels and non- SUD groups.

In summary, the current study aimed to examine the associations between three specific social supports (family support, friend support, specialist support), resilience, affect balance, and perceived stress among patients with SUD in China. The results showed that only friend support has a direct effect on perceived stress when resilience and affect balance are controlled. Secondly, we found that resilience played mediation roles in all models. Moreover, affect balance only mediates the effect of specialist support on perceived stress. These findings showed that family support, friend support, specialist support, resilience and affect balance are vital coping factors regarding reducing perceived stress among SUD patients.

\section{DATA AVAILABILITY STATEMENT}

The original contributions presented in the study are included in the article/supplementary material, further inquiries can be directed to the corresponding author/s.

\section{ETHICS STATEMENT}

The studies have been approved by the Ethics Committee of Nanjing Medical University. The patients/participants provided their written informed consent to participate in this study.

\section{AUTHOR CONTRIBUTIONS}

CY, MX, TL, and YZ drafted the manuscript. CY contributed to data analysis, results and finalized the manuscript. MX contributed to introduction, method, discussion, and finalized the manuscript. TL revised made a significant contribution to manuscript revision. YZ revised and polished the manuscript. All authors have read and approved the final manuscript.

\section{FUNDING}

This paper was supported by the Startup Foundation for Introducing Talent of NUIST; the Program B for Outstanding Ph.D. candidate of Nanjing University (202001B008); Research fund for philosophy and social science of universities in Jiangsu province (2017SJB1800). 


\section{REFERENCES}

1. Couto E Cruz C, Salom C, Maravilla J, Alati R. Mental and physical health correlates of discrimination against people who inject drugs: a systematic review. J Stud Alcohol Drugs. (2018) 79:350-60. doi: $10.15288 /$ jsad.2018.79.350

2. Deng R, Li J, Sringernyuang L, Zhang K. Drug abuse, HIV/AIDS and stigmatisation in a Dai community in Yunnan, China. Soc Sci Med. (2007) 64:1560-71. doi: 10.1016/j.socscimed.2006.12.011

3. Seib C, Whiteside E, Lee K, Humphreys J, Dao Tran TH, Chopin L, et al. Stress, lifestyle, and quality of life in midlife and older Australian women: results from the stress and the health of women study. Women Health Issues. (2014) 24:e43-52. doi: 10.1016/j.whi.2013.11.004

4. Shields GS, Sazma MA, McCullough AM, Yonelinas AP. The effects of acute stress on episodic memory: a meta-analysis and integrative review. Psychol Bull. (2017) 143:636-75. doi: 10.1037/bul0000100

5. Steptoe A, Kivimäki M. Stress and cardiovascular disease. Nat Rev Card. (2012) 9:360. doi: 10.1038/nrcardio.2012.45

6. Monat A, Lazarus RS. Stress and Coping: An Anthology (3rd ed.). New York, NY: Columbia University Press (1991). doi: 10.7312/mona 92982

7. Neale J, Brown C. we are always in some form of contact: friendships among homeless drug and alcohol users living in hostels. Health Soc Care Community. (2016) 24:557-66. doi: 10.1111/hsc.12215

8. Sherman SM, Cheng YP, Fingerman KL, Schnyer DM. Social support, stress and the aging brain. Soc Cognit Affect Neurosci. (2015) 11:1050-8. doi: $10.1093 /$ scan/nsv071

9. Wills TA, Shinar O. Measuring perceived and received social support. In: Cohen S, Underwood LG, Gottlieb BH, editors. Social Support Measurement and Intervention: A Guide for Health and Social Scientists. New York, NY: Oxford University Press (2000). doi: 10.1093/med:psych/9780195126709.003.0004

10. Lazarus RS, Folkman S. Stress, Appraisal and Coping. New York, NY: Springer (1984).

11. Cohen S, Wills TA. Stress, social support, and the buffering hypothesis. Psychol Bull. (1985) 98:310-57. doi: 10.1037/0033-2909.98. 2.310

12. Frison E, Eggermont S. The impact of daily stress on adolescents' depressed mood: the role of social support seeking through Facebook. Comp Hum Behav. (2015) 44:315-25. doi: 10.1016/j.chb.2014.11.070

13. Lee CYS, Goldstein SE, Dik BJ. The relational context of social support in young adults: links with stress and well-being. J Adult Dev. (2017) 25:25-36. doi: 10.1007/s10804-017-9271-z

14. Fletcher D, Sarkar M. Psychological resilience: a review and critique of definitions, concepts, and theory. Eur Psychol. (2013) 18:12. doi: 10.1027/1016-9040/a000124

15. Kumpfer KL. Factors and processes contributing to resilience. In: Masten, AS, Reed MGJ, editors. Resilience and Development. Boston, MA: Springer (2002), pp. 179-224.

16. Wong DFK. Differential impacts of stressful life events and social support on the mental health of mainland Chinese immigrant and local youth in Hong Kong: A resilience perspective. Br J Soc Work. (2008) 38:236-52. doi: 10.1093/bjsw/bcl344

17. Wang YW, Zhao Y, Xie SS, Wang XR, Chen Q, Xia XB. Resilience mediates the relationship between social support and quality of life in patients with primary glaucoma. Front Psychiatry. (2019) 10:5. doi: 10.3389/fpsyt.2019. 00022

18. Ghasemi A, Rahimi Foroshani A, Kheibar N, Latifi M, Khanjani N, et al. Effects of family-centered empowerment model based education program on quality of life in methamphetamine users and their families. Iran Red Crescent Med J. (2004) 16:e13375. doi: 10.5812/ircmj.13375

19. Cai WP, Pan Y, Zhang SM, Wei C, Dong W, Deng GH. Relationship between cognitive emotion regulation, social support, resilience and acute stress responses in Chinese soldiers: exploring multiple mediation model. Psychiatry Res. (2017) 256:71-8. doi: 10.1016/j.psychres.2017.06.018

20. Luthans F, Luthans K, Luthans B. Positive psychological capital: beyond human and social capital. Bus Horiz. (2004) 47:45-50. doi: 10.1016/j.bushor.2003.11.007
21. Veilleux JC, Lankford NM, Hill MA, Skinner KD, Chamberlain KD, Baker DE, et al. Affect balance predicts daily emotional experience. Pers Individ Dif. (2020) 154:109683. doi: 10.1016/j.paid.2019.109683

22. Veronese G, Pepe A, Dagdukee J, Yaghi S. Social capital, affect balance and personal well-being among teachers in Israel and Palestine. Teach. (2018) 24:951-64. doi: 10.1080/13540602.2018.1508431

23. Yang C, Zhou Y, Xia M. How resilience promotes mental health of patients with DSM-5 substance use disorder? The mediation roles of positive affect, self-esteem, and perceived social support. Front Psychiatry. (2020) 11:588968. doi: $10.3389 /$ fpsyt.2020.588968

24. Xia MF, Yang CY. The relationship among social support, self-esteem, affect balance and loneliness in individuals with substance use disorders in China. $J$ Community Psychol. (2019) 47:1269-81. doi: 10.1002/jcop.22190

25. Flueckiger L, Lieb R, Meyer AH, Witthauer C, Mata J. The importance of physical activity and sleep for affect on stressful days: two intensive longitudinal studies. Emotion. (2016) 16:488-97. doi: 10.1037/emo0000143

26. Zautra AJ, Johnson LM, Davis MC. Positive affect as a source of resilience for women in chronic pain. J Consult Clin Psychol. (2005) 73:212-20. doi: 10.1037/0022-006X.73.2.212

27. Fredrickson BL. The role of positive emotions in positive psychology - The broaden-and-build theory of positive emotions. Am Psychol. (2001) 56:21826. doi: 10.1037/0003-066X.56.3.218

28. Stroud LR, Tanofsky-Kraff M, Wilfley DE, Salovey P. The Yale Interpersonal Stressor (YIPS): Affective, physiological, and behavioral responses to a novel interpersonal rejection paradigm. Ann Behav Med. (2000) 22:204-13. doi: 10.1007/BF02895115

29. Hamama L, Ronen T, Shachar K, Rosenbaum M. Links between stress, positive and negative affect, and life satisfaction among teachers in special education schools. J Happiness Stud. (2013) 14:731-51. doi: 10.1007/s10902-0129352-4

30. Bajaj B, Pande N. Mediating role of resilience in the impact of mindfulness on life satisfaction and affect as indices of subjective well-being. Personal Individ Diff. (2016) 93:63-7. doi: 10.1016/j.paid.2015.09.005

31. Hu T, Zhang D, Wang J. A meta-analysis of the trait resilience and mental health. Pers Individ Dif. (2015) 76:18-27. doi: 10.1016/j.paid.2014. 11.039

32. Mak WW, Ng IS, Wong CC. Resilience: Enhancing well-being through the positive cognitive triad. J Couns Psychol. (2011) 58:610-7. doi: 10.1037/a0025195

33. Denovan A, Macaskill A. Stress and subjective well-being among first year UK undergraduate students. J Happiness Stud. (2016) 4:1-45. doi: 10.1007/s10902-016-9736-y

34. Affleck G, Tennen H. Construing benefits from adversity: Adaptational significance and dispositional underpinnings. J Pers. (1996) 64:899-922. doi: 10.1111/j.1467-6494.1996.tb00948.x

35. Billings DW, Folkman S, Acree M, Moskowitz JT. Coping and physical health during caregiving: The roles of positive and negative affect. J Pers Soc Psychol. (2000) 79:131-42. doi: 10.1037/0022-3514.79.1.131

36. Zimet GD, Dahlem NW, Zimet SG, Farley GK. The multidimensional scale of perceived social support. J Pers Assess. (1988) 52:30-41. doi: 10.1207/s15327752jpa5201_2

37. Chou KL. Assessing Chinese adolescents' social support: the multidimensional scale of perceived social support. Personal Individ Diff. (2000) 28:299-307. doi: 10.1016/S0191-8869(99)00098-7

38. Connor KM, Davidson JR. Development of a new resilience scale: the connor-davidson resilience scale (CD-RISC). Depress Anxiety. (2003) 18:7682. doi: 10.1002/da.10113

39. Ni MY, Li TK, Yu NX, Pang H, Chan BHY, Leung GM, et al. Normative data and psychometric properties of the Connor-Davidson Resilience Scale (CDRISC) and the abbreviated version (CD-RISC2) among the general population in Hong Kong. Q Life Res. (2015) 25:111-6. doi: 10.1007/s11136-015-1072-x

40. Yang C, Zhou Y, Cao Q, Xia M, An J. The relation-ship between selfcontrol and self-efficacy among patients with substance use disorders: resilience and self-esteem as mediators. Front Psychiatry. (2019) 10:388. doi: 10.3389/fpsyt.2019.00388

41. Watson D, Clark LA, Tellegen A. Development and validation of brief measures of positive and negative affect-The PANAS scales. J Pers Soc Psychol. (1988) 54:1063-70. doi: 10.1037/0022-3514.54.6.1063 
42. Diener E, Wirtz D, Tov W, Kim-Prieto C, Choi DW, Oishi S, et al. New well-being measures: Short scales to assess flourishing and positive and negative feelings. Soc Indic Res. (2010) 97:143-56. doi: 10.1007/s11205-0099493-y

43. Cohen S, Kamarck T, Mermelstein R. A global measure of perceived stress. $J$ Health Soc Behav. (1983) 24:385-96. doi: 10.2307/2136404

44. Yang CY, Xia MF, Han MM, Liang Y. Social support and resilience as mediators between stress and life satisfaction among people with substance use disorder in China. Front Psychiatry. (2018) 9:7. doi: 10.3389/fpsyt.2018.00436

45. American Psychiatric Association. Diagnostic and Statistical Manual of Mental Disorders (fifth edition). Washington, DC: American Psychiatric Association Publishing (2013). doi: 10.1176/appi.books.9780890425596

46. Hayes AF. Introduction to Mediation, Moderation, and Conditional Process Analysis: A Regression-Based Approach. New York, NY: Guilford Publications (2017).

47. Shrout PE, Bolger N. Mediation in experimental and nonexperimental studies: new procedures and recommendations. Psychol Methods. (2002) 7:422. doi: 10.1037/1082-989X.7.4.422

48. Martins MV, Peterson B, Almeida VM, Costa ME. Direct and indirect effects of perceived social support on women's infertility-related stress. Hum Reprod. (2011) 26:2113-21. doi: 10.1093/humrep/der157

49. Wu Q, Tsang B, Ming H. Social capital, family support, resilience and educational outcomes of Chinese migrant children. Br J Soc Work. (2014) 44:636-56. doi: 10.1093/bjsw/bcs139

50. Lakey B, Heller K. Social support from a friend, perceived support, and social problem solving. Am J Commun Psychol. (1988) 16:811-24. doi: 10.1007/BF00930894
51. Collins WA, Laursen B. Changing relationships, changing youth: Interpersonal contexts of adolescent development. J Early Adolesc. (2004) 24:55-62. doi: 10.1177/0272431603260882

52. Wolin S, Wolin S. Resilience among youth growing up in substanceabusing families. Pediatr Clin North Am. (1995) 42:415-29. doi: 10.1016/S0031-3955(16)38955-6

53. Sood A, Prasad K, Schroeder D, Varkey P. Stress management and resilience training among department of medicine faculty: a pilot randomized clinical trial. J Gene Internal Med. (2011) 26:858-61. doi: 10.1007/s11606-011-1640-x

54. Bandura A. Social Learning Theory. Englewood Cliffs, NJ: Prentice Hall (1977).

55. Xie LQ, Zhou J, Deng YL, Richmond CJ, Na J. Resilience and affect balance of empty-nest older adults with mild cognitive impairment in poor rural areas of Hunan province, China. Geriatr Gerontol Int. (2019) 19:222-7. doi: 10.1111/ggi.13601

56. Radstaak M, Geurts SAE, Brosschot JF, Cillessen AHN, Kompier MAJ. The role of affect and rumination in cardiovascular recovery from stress. Int $J$ Psychophysiol. (2011) 81:237-44. doi: 10.1016/j.ijpsycho.2011.06.017

Conflict of Interest: The authors declare that the research was conducted in the absence of any commercial or financial relationships that could be construed as a potential conflict of interest.

Copyright (C) 2021 Yang, Xia, Li and Zhou. This is an open-access article distributed under the terms of the Creative Commons Attribution License (CC BY). The use, distribution or reproduction in other forums is permitted, provided the original author(s) and the copyright owner(s) are credited and that the original publication in this journal is cited, in accordance with accepted academic practice. No use, distribution or reproduction is permitted which does not comply with these terms. 